\title{
The prognostic value of injury severity, location of event, and age at injury in pediatric traumatic head injuries
}

\author{
Jonas G Halldorsson' \\ Kjell M Flekkoy ${ }^{2}$ \\ Gudmundur B Arnkelsson ${ }^{3}$ \\ Kristinn Tomasson ${ }^{4}$ \\ Kristinn R Gudmundsson ${ }^{5}$ \\ Eirikur Orn Arnarson ${ }^{1,6}$ \\ 'Psychological Health Services, \\ Landspitali University Hospital, \\ Reykjavik, Iceland; ${ }^{2}$ Department \\ of Psychology, University of Oslo, \\ and Department of Geriatric \\ Medicine, Ulleval University Hospital, \\ Oslo, Norway; ${ }^{3}$ Department \\ of Psychology, University of Iceland, \\ Reykjavik, Iceland; ${ }^{4}$ Administration \\ of Occupational Safety and Health, \\ Reykjavik, Iceland; ${ }^{5}$ Department \\ of Neurosurgery, Landspitali \\ University Hospital, Reykjavik, Iceland; \\ ${ }^{6}$ Faculty of Medicine, University \\ of Iceland, Reykjavik, Iceland
}

\begin{abstract}
Aims: To estimate the prognostic value of injury severity, location of event, and demographic parameters, for symptoms of pediatric traumatic head injury (THI) 4 years later.

Methods: Data were collected prospectively from Reykjavik City Hospital on all patients age 0-19 years, diagnosed with THI $(n=408)$ during one year. Information was collected on patient demographics, location of traumatic event, cause of injury, injury severity, and ICD-9 diagnosis. Injury severity was estimated according to the Head Injury Severity Scale (HISS). Four years post-injury, a questionnaire on late symptoms attributed to the THI was sent.

Results: Symptoms reported were more common among patients with moderate/severe THI than among others $(\mathrm{p}<0.001)$. The event location had prognostic value $(\mathrm{p}<0.05)$. Overall, $72 \%$ of patients with moderate/severe motor vehicle-related THI reported symptoms. There was a curvilinear age effect $(\mathrm{p}<0.05)$. Symptoms were least frequent in the youngest age group, 0-4 years, and most frequent in the age group 5-14 years. Gender and urban/rural residence were not significantly related to symptoms.
\end{abstract}

Conclusions: Motor vehicle related moderate/severe THI resulted in a high rate of late symptoms. Location had a prognostic value. Patients with motor vehicle-related THI need special consideration regardless of injury severity.

Keywords: follow-up, pediatric, symptoms, traumatic head injury

\section{Introduction}

Traumatic head injury (THI) leading to brain damage is a major cause of death and disability in childhood and youth (Kraus et al 1986; Rivara 1994; Arnarson and Halldorsson 1995; Emanuelson and Wendt 1997; Jennett 1998; Halldorsson et al 2007).

Most children receive THI at some time. Many minor injuries may never receive attention from healthcare personnel. Fortunately, most recorded THI are minimal or mild with fast recovery and no apparent complications (Kraus and McArthur 1996). Nonetheless, every THI may potentially lead to serious damage (Jennett 1998). Estimating the severity of THI in the acute and sub-acute phase is therefore critical, but can be problematic especially in infancy and early childhood, when clinical signs may be less marked and responses to trauma may differ from those of older individuals (Bernardi et al 1993; Dietrich et al 1993; Quayle et al 1997; Greenes and Schutzman 1998; Savitsky and Votey 2000; Schutzman et al 2001). Pediatric THI, irrespective of cause, and even if considered mild in the acute phase, may have debilitating long-term consequences (Jennett 1998).

Research has shown that force of impact is positively correlated with injury severity. High velocity motor vehicle-related collisions are among the leading causes of severe and fatal injuries in childhood (Vane and Shackford 1995; Boswell et al 1996; DiMaggio and Durkin 2002). Infants and young children are at a relatively high risk 
of minimal and mild THI caused by falling (Arnarson and Halldorsson 1995; Halldorsson et al 2007). Evidence suggests that craniofacial injuries in young children caused by slipping, tripping, or falling most often take place at home and are rarely serious or fatal (Chang and Tsai 2007). However, THI involving relatively mild impact, eg, caused by stumbling and falling, or falling from a table, may lead to moderate or severe brain injury, especially in infants and young children (Emanuelson and Wendt 1997).

The previously held assumption that young children recover better from brain injury than older children, because of developmental plasticity has not been substantiated. On the contrary, early brain damage disrupts normal maturation and development, and neuronal plasticity may not always promote an optimal outcome (Chapman and McKinnon 2000; Giza and Prins 2006).

Recent research findings have indicated that development of infants and young children may be more adversely affected by brain injury than the development of older children and adolescents (Taylor and Alden 1997; Anderson et al 2000; Eslinger et al 2004). Recovery of intelligence quotient (IQ) in young children 3-7 years old who suffered severe brain injury has been found to be minimal 12 months post-injury. Recovery of IQ in older children was better and similar to that of adults (Anderson et al 2000). In children 6-12 years old with moderate and severe brain injury, sequelae related to cognition had not resolved four years post-injury (Yeates et al 2004).

Pediatric traumatic brain injuries can have significant and persistent sequelae, affecting intellectual and other cognitive functions, prefrontal executive functioning, social adaptation and behavior, academic performance, emotion, and personality (Levin et al 1993; Anderson et al 2004; Eslinger et al 2004; Ewing-Cobbs et al 2006; Max et al 2006).

The social and cognitive functions of those who have suffered childhood brain injuries may even decline with age, as indicated by repeated assessments post-injury (Jonsson et al 2004; Yeates et al 2004; Levine et al 2005). Some sequelae of early brain injury may not be fully manifested until adolescence or early adulthood (Brooke 1988; Eslinger et al 1992).

Boys are at greater risk of sustaining THI than girls (Halldorsson et al 2007). Due to greater adaptability associated with the female gender in infancy and early childhood (Prior et al 1993), girls may make a better recovery from early THI than boys. Groswasser and colleagues (1998) found that in a group of patients with severe brain injury aged 5-65 years old, female patients had a better predicted outcome at the time of discharge from an in-patients rehabilitation program than male patients.
Both in the US and in Iceland, the pediatric accident mortality rate has been found to be higher in rural compared with urban areas, and motor vehicle crashes have been a common cause of fatal injuries (Vane and Shackford 1995; Stefansdottir and Mogensen 1997; Eberhardt and Pamuk 2004). In addition, looking at the incidence of fatal THI in Iceland, specifically, its rate seems to be higher in rural areas than in the Reykjavik area (Halldorsson et al 2007). This may suggest that THI resulting from high force on impact is more common in rural areas than in the Reykjavik area, leading to more serious injuries and more long-term sequelae. The Icelandic environment is diverse, with one major urban area, and rural areas with small towns, villages, and farmland. The low population density, and thus significant distance to healthcare providers in rural areas, increases the likelihood of delayed emergency services (Olafsson and Sigurdsson 2000).

The present study was part of a larger, prospective, longitudinal research project, aimed at assessing the nationwide incidence and long-term cognitive and socioeconomic consequences of early THI.

In the present study, our aim was to estimate the prognostic value of injury severity, location of event, age at injury, gender and urban/rural residence, for reported symptoms of THI 4 years post-injury. An additional aim was to obtain information on what characterizes young THI patients who need specialized intervention in the sub-acute phase and long-term follow-up.

\section{Material and methods}

\section{Patients}

This prospective study comprised all 408 children and adolescents, 0-19 years old, consecutively diagnosed with THI, International Classification of Diseases 9 (ICD-9) 850-854 (WHO 1977), at Reykjavik City Hospital during the year from April 15, 1992 to April 14, 1993. Of the 408 patients, 343 were treated at the emergency unit of Reykjavik City Hospital and subsequently discharged, while 61 were hospitalized. Four received fatal THI.

By law, Icelandic adolescents take on most of the responsibilities of adults at 18 years of age, but we decided on an upper age limit of 19 rather than 17 years. The majority of Icelandic adolescents do not complete grammar school or trade school until age 20, are living with their parents, and rely on their support, almost certainly when traumatic events occur.

\section{Procedures}

At the time of the study, the only neurosurgical unit and the only computed tomography (CT) scanners in Iceland 
were located in Reykjavik. Practically all patients in Iceland diagnosed with or suspected of moderate or severe THI (ICD-9 851-854) were brought to the Reykjavik City Hospital by ambulance, helicopter, airplane, or by sea. When the diagnosis and degree of severity were uncertain, expert advice was available by telephone and transport to Reykjavik encouraged. Medical services were provided for minimal or mild THI by local hospitals and healthcare centers. The emergency unit of Reykjavik City Hospital served mainly the Reykjavik area. The study group included a nationwide sample of patients $0-19$ years with moderate/severe THI, and a sample of same age patients with minimal/mild THI, the majority living in the Reykjavik area.

Information was collected on the age, gender, and urban/rural residence of THI patients, as well as the location of the traumatic event, cause of injury, injury severity, and ICD-9 diagnosis. The primary author ascertained new admissions daily, through information provided by neurosurgeons, other hospital personnel, and written and computerized hospital records.

Data were collected from the Icelandic Death Register (Statistics Iceland 2001) on patients who received fatal THI during the same period. Included were patients who died after being admitted to hospital and those who died at the scene or during transport to hospital.

We sent a short questionnaire 4 years post-injury to the custodial parents of the THI patients, or to the patients themselves if they had reached 18 years of age. The patient version of the questionnaire included three questions. Question one: Do you still have any symptoms that you attribute to the traumatic head injury you suffered 4 years ago? Answer "Yes" or "No". Question two: If the answer to question one is "Yes", please describe those symptoms in a couple of sentences. Question three: Have you sought professional advice regarding those symptoms. Answer "Yes" or "No". The focus in the present study is on the results of answers to Question one.

The overall response rate to the questionnaire was $60 \%$, comparable across sub-groups of age and gender. Approximately $58 \%$ of patients with minimal/mild THI and $66 \%$ of those with moderate/severe THI answered the questionnaire. Responders and nonresponders were comparable in age and gender distribution.

Four years post-injury, data were obtained from the State Social Security Institute (SSSI), to see if and why any of the 405 patients had been added to the list of children and adolescents receiving benefits intended for the disabled or chronically ill, since the time of injury. Patients' identification numbers were sent to the SSSI without any reference to
THI. The data from the SSSI could not be linked to patients' names or identification numbers.

\section{Classifications}

All patients were classified according to ICD-9 (WHO 1977) diagnostic codes 850-854: ICD-9 850 denotes concussion; 851 cerebral laceration and contusion; 852 subarachnoid, subdural, and extradural hemorrhage following injury; 853 other and unspecified intracranial hemorrhage following injury; and 854 intracranial injury of unspecified nature. Corresponding diagnostic codes can be found in ICD-10, S06.0-S06.9 (WHO 1992).

Patients with more than one ICD-9 diagnosis were included if at least one of the diagnoses was in the 850-854 range. Patients with more than one 850-854 diagnosis were identified according to the most serious one.

Physicians diagnosed concussion following THI, based on clinical symptoms, such as loss of or reduced consciousness, confusion, dizziness, somnolence, nausea, or amnesia. ICD-9 851-853 diagnoses were made by neurosurgeons, based on cerebral CT findings.

In accordance with the Scandinavian Guidelines for Initial Management of Minimal, Mild, and Moderate Head Injuries (SNC Guidelines) (Ingebrigtsen et al 2000), concussed patients with Glasgow Coma Scale (GCS) (Teasdale and Jennett 1974) score 15, no loss of consciousness (LOC) and no signs of skull fracture or other complications, were not admitted to hospital, but discharged from the emergency unit with head injury instructions. Concussed patients with GCS scores lower than 15, or LOC, or amnesia, or signs of skull fracture, or other complications were hospitalized. Referral to cerebral CT was based on neurosurgical consultation and expert opinion on injury severity and complications.

We estimated injury severity according to the Head Injury Severity Scale (HISS) (Stein and Spettell 1995), and the SNC Guidelines (Ingebrigtsen et al 2000). Concussion with GCS score 15 , no LOC, and no amnesia was classified as minimal THI. Concussion with GCS 14 or 15 , brief ( $<5$ minutes) LOC or amnesia, or impaired alertness or memory, was categorized as mild THI. The criteria for moderate THI were GCS 9-13, or LOC $\geq 5$ minutes, or focal neurological deficit, or abnormal cerebral CT, or skull fracture. GCS 3-8 was classified as severe THI.

As the variables "Cause of THI" and "Where the THI took place" were related, we made a new variable based on the two, called "Location of event". This variable had three categories: In the first category, "Home", THI occurred at home, mainly caused by fall; in the second category, "Outside home", THI 
occurred outside the home, for example in playgrounds, schools, sports facilities, including all causes other than motor vehicle or heavy machinery; and in the third category, "Motor vehicle", THI caused by motor vehicles and heavy machinery.

A distinction was made between patients living in the greater Reykjavik area (total population 0-19 years: 47,327), and patients living in rural areas and small towns and villages in other parts of Iceland (total population 0-19 years: 38,419). Reykjavik with its surrounding towns and suburbs is the only major urban area in Iceland. The largest town outside the Reykjavik area was Akureyri with a total population $0-19$ years of 4,903 .

The study group was divided according to gender. Boys were $58 \%$ of the total group of patients, $57 \%$ in the minimal/ mild THI group and $63 \%$ in the moderate/severe group.

We converted answers to Question two of the questionnaire, Description of symptoms, into Glasgow Outcome Scale (GOS) scores (Jennett and Bond 1975), with criteria modified for infants and children (Ewing-Cobbs et al 1998; Barlow et al 2005): 1) Good Outcome: Return to age appropriate or pre-injury levels of functioning, even though there may be minor neurological or psychological deficits; 2) Moderate Disability: Significant reduction in cognitive functioning from estimated premorbid levels and personality changes; and/or motor deficits interfering with activities of daily living; and/or referral to outpatient rehabilitation or special education therapies; 3) Severe Disability: (Conscious but disabled). Cognitive functioning in the deficient range; and or severe motor deficits. Patient depends upon others for daily support due to mental or physical disability or both; 4) Persistent Vegetative State: Patient exhibits no obvious cortical function; and 5) Death.

\section{Statistical analysis}

Binary logistic regression was used to predict symptoms of pediatric THI 4 years post-injury. The full model contained the five plausible main effects, injury severity, location of event, age at injury, gender, and urban/rural residence. Backward elimination from the full model removed two nonsignificant effects, gender and urban/rural residence, leading to a model of the three remaining main effects. Statistical significance was calculated with chi-squares based on likelihood ratio. Age was coded as a second-degree polynomial allowing for a curvilinear effect for age. SPSS for Windows, Release 15.0.0 (SPSS Inc., Chicago, IL, USA), was used for the statistical analyses.

\section{Ethics}

The Icelandic Data Protection Commission, the Icelandic Medical Ethics Committee, and the medical directors concerned approved the protocol. Permission was obtained from Statistics Iceland regarding use of data from the Icelandic Death Register.

\section{Results}

The cross-classification by injury severity, location of event, age, and symptoms reported, resulted in uneven distribution of data. This led to sparse data in some subgroups, particularly for minimal/mild THI involving a motor vehicle. Overall, 49 patients suffered moderate/severe THI, about $12 \%$ of the total sample (Table 1 ).

The model adopted, containing the three main effects, severity of injury, location of event, and age at injury, was statistically significant $\left(\chi^{2}=63.9, \mathrm{df}=3, \mathrm{p}<0.001\right)$. Figure 1 gives the fitted values of the model.

Symptoms reported 4 years post-injury were more common among patients with moderate/severe THI than among those with $\mathrm{minimal} / \mathrm{mild} \mathrm{THI}$ (odds ratio $[\mathrm{OR}]=8.3,95 \%$ confidence interval $[\mathrm{CI}]=3.4-20.3, \chi^{2}=20.9$, df $=1, \mathrm{p}<0.001$ ).

Controlling for the two other effects of the main model, injury severity and age at injury, symptoms reported were related to location of event $\left(\chi^{2}=6.5\right.$, $\left.\mathrm{df}=2, \mathrm{p}=0.039\right)$. Symptoms reported were least likely when injury took place at home, but increased when it took place outside the home $(\mathrm{OR}=2.4,95 \% \mathrm{CI}=0.5-10.5)$, and were most likely when motor vehicles and heavy machinery were involved $(\mathrm{OR}=7.0,95 \%$ CI 1.4-35.7).

Symptoms were least likely in the youngest age group ( $0-4$ years), and substantially more likely in the 5-9 years and 10-14 years age groups than in the 15-19 years age group, resulting in a curvilinear age trend, $\chi^{2}=7.5, \mathrm{df}=2$, $\mathrm{p}=0.024$, as depicted in Figure 1 .

We neither found an effect for gender $\left(\chi^{2}=0.003\right.$, $\mathrm{df}=1, \mathrm{p}=0.957)$ nor $\mathrm{urban} /$ rural residence $\left(\chi^{2}=1.7, \mathrm{df}=1\right.$, $\mathrm{p}=0.197)$.

One two-way interaction was significant: severity of injury by location of event $\left(\chi^{2}=6.1, \mathrm{df}=2, \mathrm{p}=0.047\right)$. This interaction suggested that minimal/mild THI related to motor vehicles had fewer sequelae than minimal/mild THI from other causes outside the home, which we found implausible. Despite nominal significance, considering the few patients in the minimal/mild motor vehicle subgroup, as well as the theoretical intractability, we regarded the interaction as an instance of overfitting of random variations in the somewhat sparse data.

No three-way interactions were significant.

Of 19 patients who reported symptoms following minimal/mild THI, 13 (68\%) complained of chronic headache. The remaining six (32\%) reported physical, as well as 
Table I Number of patients diagnosed with traumatic head injury (THI) (ICD-9 850-854) by age at injury, injury severity, location of event, and by symptoms, four years post-injury

\begin{tabular}{|c|c|c|c|c|c|}
\hline Type of THI & $0-4$ years & 5-9 years & $10-14$ years & $15-19$ years & Total \\
\hline \multicolumn{6}{|l|}{ Moderate/severe THI ${ }^{a}$} \\
\hline At home - symptom & 0 & 1 & 0 & 0 & 1 \\
\hline At home - no symptom & 11 & I & I & 0 & 13 \\
\hline Outside home - symptom & 0 & 1 & 4 & 1 & 6 \\
\hline Outside home - no symptom & 0 & 4 & 4 & 3 & 11 \\
\hline Motor vehicle - symptom or fatal & 2 & 3 & 3 & 5 & 13 \\
\hline Motor vehicle - no symptom & 1 & 0 & I & 3 & 5 \\
\hline Total number with symptoms & 2 & 5 & 7 & 6 & 20 \\
\hline Total number injured & 14 & 10 & 13 & 12 & 49 \\
\hline \multicolumn{6}{|l|}{ Minimal/mild THI } \\
\hline At home - symptom & 2 & 0 & 0 & 0 & 2 \\
\hline At home - no symptom & 114 & 17 & I & 3 & 135 \\
\hline Outside home - symptom & 0 & 5 & 9 & 3 & 17 \\
\hline Outside home - no symptom & 52 & 49 & 61 & 34 & 196 \\
\hline Motor vehicle - symptom & 0 & 0 & 0 & 0 & 0 \\
\hline Motor vehicle - no symptom & 1 & 1 & 3 & 4 & 9 \\
\hline Total number with symptoms & 2 & 5 & 9 & 3 & 19 \\
\hline Total number injured & 169 & 72 & 74 & 44 & 359 \\
\hline
\end{tabular}

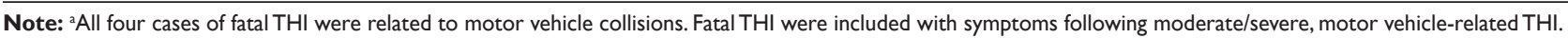

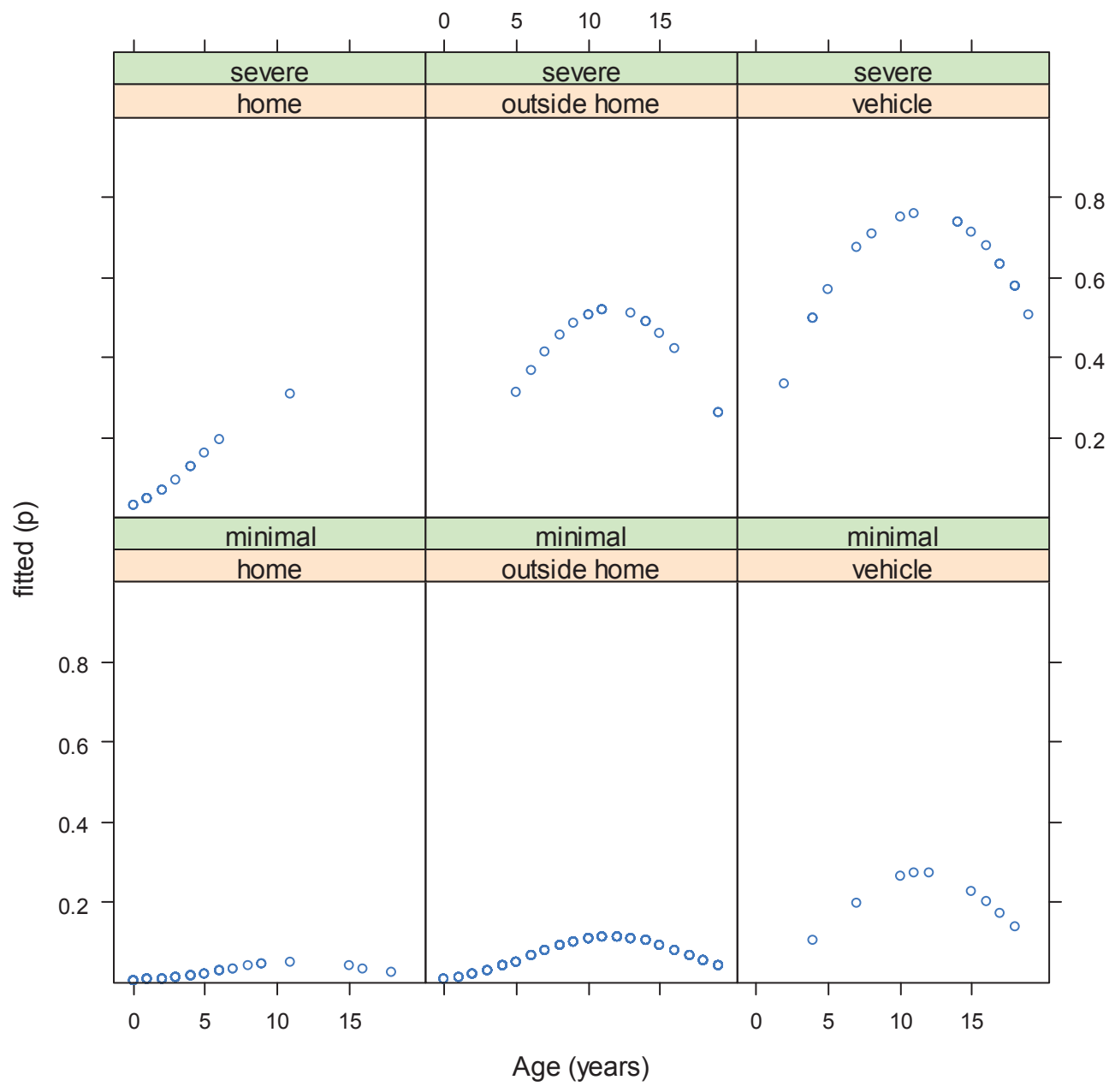

Figure I The fitted values of the model of the three main effects, traumatic head injury severity, location of event, and age at injury. 
cognitive and adaptive symptoms, such as chronic headache, sleep disorder, motor problems, lack of concentration, problems with verbal expression, and obsessive-compulsive traits. Professional advice about symptoms had been sought in eight (42\%) cases. According to GOS, 14 (74\%) patients had a good outcome and five (26\%) had moderate disability. Of the total group of patients with minimal/mild THI $(n=359)$, all but five (1\%) had a good outcome.

In the group of 20 patients who reported symptoms following moderate/severe THI, three (15\%) had physical complaints limited to chronic headache or loss of hearing. The rest of the patients reported a variety of physical, cognitive, and adaptive symptoms, such as sleep disorder, epileptic episodes, tic disorder and stuttering, motor problems and indications of hemiparesis, general slowness, lack of concentration, attention and stamina, problems with memory and verbal expression, change of personality and temper, lack of self-restraint, dependence, emotional problems, and depression. Fifteen (75\%) patients had sought professional advice because of their symptoms. According to the GOS, five $(25 \%)$ of the 20 patients had a good outcome, nine $(45 \%)$ had moderate disability, two $(10 \%)$ had severe disability, and four $(20 \%)$ died. In the total group of 49 patients with moderate/severe THI, 34 (69\%) had a good outcome, while $15(31 \%)$ had moderate/severe disability or died.

\section{Discussion}

Results support the validity of symptoms reported, with more severe THI resulting in greater frequency of symptoms than less severe THI. The high risk of symptoms reported 4 years post-injury in moderate/severe THI, outside the home and motor vehicle related, indicates a need for specialized intervention and assessment in the sub-acute phase and subsequent follow-up for patients at risk of long-term sequelae.

We included fatal THI in the statistical analysis so that we would not run the risk of underestimating the severity of THI among children and adolescents. While admittedly not a reported symptom, death may be seen as an indicator of severe injury sequelae. For prevention purposes, it is important to note that all cases of fatal THI were related to high velocity motor vehicle collisions.

In line with previous research (Jennett 1998; Yeates and Taylor 2005), the present findings suggest that minimal/mild THI may lead to long-term sequelae. Nineteen $(5 \%)$ of the 359 patients with minimal/mild THI reported late sequelae, stressing the need for instructions to parents on post concussion symptoms. Although assessment of THI severity in the acute phase provides valuable information regarding prognosis, based on well established criteria such as the HISS (Stein and
Spettell 1995), it may not fully address the extent of neurological disruption and late neuropsychological sequelae.

Symptoms reported were positively related to the location of event 4 years post-injury. We suggest that this increase in symptoms reflects in part the increased force of impact, resulting in more extensive injuries. We propose that the majority of THI at home are low impact injuries, that there is an increase in force when THI takes place outside the home, for example in playgrounds, schools, and sport facilities, and that highest impact THI is most often related to motor vehicles and heavy machinery.

Previous research (Vane and Shackford 1995; Boswell et al 1996; DiMaggio and Durkin 2002) has shown that motor vehicle collisions are a common cause of serious brain injury, and that THI in young children caused by slipping, tripping or falling in the home is most often minimal or mild (Chang and Tsai 2007). Obviously, there are exceptions; even a relatively low fall in the home, the most common cause of THI in early age, may cause cerebral contusion or hemorrhage in infants and toddlers (Emanuelson and Wendt 1997). In our study, three 0-3 year old children were diagnosed with ICD-9 851-854, caused by falls in the home. These findings are important for injury prevention purposes.

There was a significant curvilinear age effect $(\mathrm{p}<0.05)$, where symptoms reported were least likely in the youngest age group 0-4 years old, and most likely in the two age groups 5-9 and 10-14 years.

The relatively few cases of symptoms reported among children in the youngest age group, sustaining moderate/ severe THI, were unanticipated, because the number of moderate/severe THI was identical across the four age groups, as was the parents' response rate to the questionnaire. Young children have not been found to recover better from brain injury than older children (Taylor and Alden 1997; Anderson et al 2000; Chapman and McKinnon 2000; Eslinger et al 2004; Giza and Prins 2006). In the present study, young children may, however, have received less extensive neurological injury than older ones. The actual severity may not have been fully captured in the injury severity variable. A later followup study is indicated to help clarify the age effect.

It may be speculated that other reasons than injury severity contribute to the low frequency of symptoms reported in the 0-4 year old. For example, healthcare professionals have more difficulty detecting THI severity in infants and young children than in older ones in the acute and sub-acute phase (Bernardi et al 1993; Dietrich et al 1993; Quayle et al 1997; Greenes and Schutzman 1998; Savitsky and Votey 2000; Schutzman et al 2001). Parents may also have greater 
difficulty discerning THI sequelae in infants and young children, than in older children and adolescents. Professional awareness regarding the sequelae of moderate/severe THI in infants and young children is indicated, as is support and instructions to parents.

In this vein, it may be added that 4 years post-injury, six of the younger children had been added to the SSSI records because of hyperkinetic disorder, conduct disorder, social adaptation disorder, sleep disorder, or tic disorder, without referring to the previous THI. Misdiagnosis may lead to less than optimal intervention and outcome.

Controlling for injury severity, location of event, and age at injury, we did not establish any relationship between gender and symptoms reported. In particular, we did not find evidence for the earlier finding that females recover better than males from THI (Groswasser et al 1998). While THI is more common in boys than girls, the results indicate that given the same severity, location of event and (possibly) age, similar specialized intervention and follow-up may be recommended regardless of patient gender.

In our study group, no significant differences were found between the Reykjavik area and rural areas in the incidence of moderate/severe THI (Halldorsson et al 2007), and in the present study, symptoms reported 4 years post-injury were not significantly related to urban/rural residence. This suggests that specialized intervention and follow-up must be accessible, in both urban and rural areas.

\section{Limitations and future directions}

The validity of the dependent variable, symptoms reported, may be open to question. In addition, there is the possibility of response bias. However, the content analysis of symptoms, and classification of symptoms according to GOS scores, suggesting that more complex symptoms and more serious outcomes on GOS relate to the more serious THI, lends increased support to the validity of the dependent measure.

A larger number of patients in some of the subgroups, such as minimal/mild, motor vehicle-related THI, would have been preferable for the statistical analyses. The proportional distribution of participants in the study, however, reflected the incidence of pediatric THI in Iceland. A validation of the present results in a larger sample is indicated.

There are uncertainties related to the classification of THI severity in the acute phase. For example, accurate information may be lacking with respect to length of loss of consciousness, and the extent of neurological injury may at times be underestimated. However, the HISS was applicable to the present data according to the SNC Guidelines in a consistent manner.

The wide age range makes interpreting our data more complicated, but at the same time, the age range and injuries of different severity are the strength of the study.

A follow-up of the study group is presently being planned, as well as a detailed questionnaire, and more objective measures of outcome. A large control group will be included. A subgroup of 50 patients with the more severe THI will undergo neuropsychological evaluation. The study may provide additional information regarding the late effects of early THI, its relationship to age at injury, and early prognostic symptoms.

\section{Conclusions}

The present study indicates the long-term consequences of pediatric THI. Our findings suggest that minimal/mild THI may in some cases lead to late sequelae, stressing the need for instructions to parents on post concussion symptoms. Because of increased risk of late sequelae, children and adolescents diagnosed with moderate/severe THI should receive specialized evaluation in the sub-acute phase with respect to their need for intervention and long-term followup. Patients with minimal/mild and moderate/severe motor vehicle-related THI need special consideration. Further research is needed on late sequelae of moderate/severe THI in the youngest age group, 0-4 years. Misdiagnosis may lead to less than optimal intervention and outcome.

\section{Acknowledgments}

The authors are grateful for support, assistance, and information provided by Statistics Iceland, Icelandic Directorate of Health, and Landspitali University Hospital. The study was funded in part with grants from the European Council, Nordisk Forskeruddannelsesakademi NorFA, the NATO Science Foundation, The University of Oslo, Reykjavik City Hospital, and the Icelandic Accident Prevention Council.

\section{References}

Anderson V, Catroppa C, Morse S, et al. 2000. Recovery of intellectual ability following traumatic brain injury in childhood: impact of injury severity and age at injury. Pediatr Neurosurg, 32:282-90.

Anderson VA, Morse SA, Catroppa C, et al. 2004. Thirty month outcome from early childhood head injury: a prospective analysis of neurobehavioural recovery. Brain, 127:2608-20.

Arnarson EO, Halldorsson JG. 1995. Head trauma among children in Reykjavik. Acta Paediatr, 84:96-9.

Barlow KM, Thomson E, Johnson D, et al. 2005. Late neurologic and cognitive sequelae of inflicted traumatic brain injury in infancy. Pediatrics, $116: 174-85$

Bernardi B, Zimmermann RA, Bilaniuk LT. 1993. Neuroradiologic evaluation of pediatric craniocerebral trauma. Top Magn Reson Imaging, 5:161-73. 
Boswell WC, Boyd CR, Schaffner D, et al. 1996. Prevention of pediatric mortality from trauma: Are current measures adequate? South Med J, 89:218-20.

Brooke OG. 1988. Delayed effects of head injuries in children. $\mathrm{Br}$ Med $J, 296: 948$.

Chang LT, Tsai MC. 2007. Craniofacial injuries from slip, trip, and fall accidents of children. $J$ Trauma, 63:70-4.

Chapman SB, McKinnon L. 2000. Discussion of developmental plasticity: Factors affecting cognitive outcome after pediatric traumatic brain injury. J Commun Disord, 33:333-44.

Dietrich AM, Bowman MJ, Ginn-Pease ME, et al. 1993. Pediatric head injuries: Can clinical factors reliably predict an abnormality on computed tomography? Ann Emerg Med, 22:1535-40.

DiMaggio C, Durkin M. 2002. Child pedestrian injury in an urban setting: Descriptive epidemiology. Acad Emerg Med, 9:54-62

Eberhardt MS, Pamuk ER. 2004. The importance of place of residence: Examining health in rural and nonrural areas. Am J Public Health, 94:1682-6.

Emanuelson I, Wendt LV. 1997. Epidemiology of traumatic brain injury in children and adolescents in southwestern Sweden. Acta Paediatr, $86: 730-5$.

Eslinger PJ, Flaherty-Craig CV, Benton AL. 2004. Developmental outcomes after early prefrontal cortex damage. Brain Cogn, 55:84-103.

Eslinger PJ, Grattan LM, Damasio H, et al. 1992. Developmental consequences of frontal lobe damage. Arch Neurol, 49:764-9.

Ewing-Cobbs L, Kramer L, Prasad M, et al. 1998. Neuroimaging, physical, and developmental findings after inflicted and noninflicted traumatic brain injury in young children. Pediatrics, 102:300-7.

Ewing-Cobbs L, Prasad MR, Kramer L, et al. 2006. Late intellectual and academic outcomes following traumatic brain injury sustained during early childhood. J Neurosurg: Pediatrics, 105:287-96.

Giza CC, Prins ML. 2006. Is being plastic fantastic? Mechanisms of altered plasticity after developmental traumatic brain injury. Dev Neurosci, 28:364-79.

Greenes DS, Schutzman SA. 1998. Occult intracranial injury in infants. Ann Emerg Med, 32:680-6.

Groswasser Z, Cohen M, Keren O. 1998. Female TBI patients recover better than males. Brain Inj, 12:805-8.

Halldorsson JG, Flekkoy KM, Gudmundsson KR, et al. 2007. Urban-rural differences in pediatric traumatic head injuries: A prospective nationwide study. Neuropsychiatr Dis Treat, 3:935-41.

Ingebrigtsen T, Romner B, Kock-Jensen C. 2000. Scandinavian guidelines for the initial management of minimal, mild, and moderate head injuries. J Trauma, 48:760-6.

Jennett B. 1998. Epidemiology of head injury. Arch Dis Child, 78:403-6.

Jennett B, Bond M. 1975. Assessment of outcome after severe brain damage. A practical scale. Lancet, 1:480-4.

Jonsson CA, Horneman G, Emanuelson I. 2004. Neuropsychological progress during 14 years after severe traumatic brain injury in childhood and adolescence. Brain Inj, 18:921-34.

Kraus JF, Fife D, Cox P, et al. 1986. Incidence, severity, and external causes of pediatric brain injury. Am J Dis Child, 140:687-93.
Kraus JF, McArthur DL. 1996. Epidemiologic aspects of brain injury. Neurol Clin, 14:435-50.

Levin HS, Culhane KA, Mendelsohn D, et al. 1993. Cognition in relation to magnetic resonance imaging in head-injured children and adolescents. Arch Neurol, 50:897-905.

Levine SC, Kraus R, Alexander E, et al. 2005. IQ decline following early unilateral brain injury: A longitudinal study. Brain Cogn, 59:114-123.

Max JE, Levin HS, Schachar RJ, et al. 2006. Predictors of personality change due to traumatic brain injury in children and adolescents six to twenty-four months after injury. J Neuropsychiatry Clin Neurosci, 18:21-32.

Olafsson G, Sigurdsson JA. 2000. Out-of-hours service in rural areas. An observational study of accessibility, attitudes and quality standards among general practitioners in Iceland. Scand J Prim Health Care, 18:75-9.

Prior M, Smart D, Sanson A, et al. 1993. Sex differences in psychological adjustment from infancy to 8 years. J Am Acad Child Adolesc Psychiatry, 32:291-304.

Quayle KS, Jaffe DM, Kuppermann N, et al. 1997. Diagnostic testing for acute head injury in children: When are head computed tomography and skull radiographs indicated? Pediatrics, 99, e11 [online]. Accessed 1 October 2006. URL: http://www.pediatrics.org/cgi/content/full/99/5/e11.

Rivara FP. 1994. Epidemiology and prevention of pediatric traumatic brain injury. Pediatr Ann, 23:12-7.

Savitsky EA, Votey SR. 2000. Current controversies in the management of minor pediatric head injuries. Am J Emerg Med, 18, 96-100.

Schutzman SA, Barnes P, Duhaime AC, et al. 2001. Evaluation and management of children younger than two years old with apparently minor head trauma: Proposed guidelines. Pediatrics, 107:983-93.

Statistics Iceland. 2001. Danarmeinaskra [The Icelandic death register]. Reykjavik: Statistics Iceland.

Stefansdottir A, Mogensen B. 1997. Epidemiology of childhood injuries in Reykjavik 1974-1991. Scand J Prim Health Care, 15:30-4.

Stein SC, Spettell C. 1995. The Head Injury Severity Scale (HISS): a practical classification of closed-head injury. Brain Inj, 9:437-44.

Taylor HG, Alden J. 1997. Age-related differences in outcomes following childhood brain insults: an introduction and overview. J Int Neuropsychol Soc, 3:555-67.

Teasdale G, Jennett B. 1974. Assessment of coma and impaired consciousness. Lancet, ii:81-4.

Vane DW, Shackford SR. 1995. Epidemiology of rural traumatic death in children: A population-based study. J Trauma, 38:867-70.

[WHO] World Health Organization. 1977. International classification of diseases (9th ed.). Geneva: World Health Organization.

[WHO] World Health Organization. 1992. International statistical classification of diseases and related health problems (10th ed.). Geneva: World Health Organization.

Yeates KO, Swift E, Taylor HG, et al. 2004. Short- and long-term social outcomes following pediatric traumatic brain injury. J Int Neuropsychol Soc, $10: 412-26$.

Yeates KO, Taylor HG. 2005. Neurobehavioural outcomes of mild head injury in children and adolescents. Pediatr Rehab, 8:5-16. 